Finally, Clark and Hassink announced that free access has been granted to the archives of fourteen core mathematics journals for the years from 1995 through four years before the present day. Access to back issues is indeed critical, and we strongly believe that all research papers should be made freely available long before copyright expires. The shorter the delay the better, of course, but we consider four years a defensible choice, compatible with the subscription model for journal publishing. The AMS's experience with a five-year window shows that such a move is financially viable. We hope that Elsevier's announcement is just the first step and that expansion to the full set of mathematical journals and the period before 1995 will be announced soon. ${ }^{19}$ We also hope that this is not just a temporary measure. A binding commitment not to revoke access in the future would be reassuring on that point.

\section{Moving Forward}

While the mathematical literature itself is a treasure, the current system of scholarly publishing is badly broken. Elsevier is the largest and, in our view, the most egregious example of what is wrong. We hope many readers will agree with us that by

${ }^{19}$ All three journals discussed here began publishing in the 1960s. The issues before 1995 are currently available from Elsevier online but remain behind their paywall. choosing to withdraw our cooperation from Elsevier, we are sending a valuable message to them and to the scholarly publishing industry more broadly. Please consider joining the movement at http://thecostofknowledge. com.

What is our vision for the future? The mathematical community needs a period of experimentation and healthy competition, in which a variety of publishing models can flourish and develop. Possibilities include various approaches to open access publishing, ${ }^{20}$ refereed journals tightly integrated with the arXiv or similar servers, increased reliance on nonprofit publishers, hybrid models in which community-owned journals subcontract their operations to commercial publishers, commercially owned journals with reasonable prices and policies, etc. It is too early to predict the mix of models that will emerge as the most successful. However, any publisher that wants to be part of this mix must convince the community that they oversee peer review with integrity, that they aid dissemination rather than hinder it, and that they work to make high-quality mathematical literature widely available at a reasonable price.

Let's work together to foster good practices and build better models. The future of mathematics publishing is in our hands.

${ }^{20}$ For example, based on publication charges or on spon-

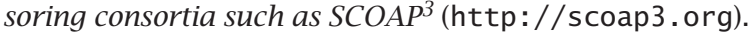

\title{
Elsevier's Response to the Mathematics Community
}

\section{Laura Hassink and David Clark}

During the last months we have spoken to many people in the community about the move by Timothy Gowers and some colleagues to declare their wish not to work with Elsevier and the subsequent boycott movement.

At the end of this article we summarize how we are responding to the feedback from the community and the very specific steps that we are taking. But we would first like to address the concerns raised and some of the arguments.

We are the leading journal publisher in scientific publishing and so will attract criticism that is

Laura Hassink is Senior Vice President, Publishing, Elsevier. Her email address is 1. hassink@e1sevi er. com.

David Clark is Senior Vice President, Publishing, Elsevier. His email address is david. clark@e1sevier.com.

DOI: http://dx.doi.org/10.1090/noti853 directed at publishing as a whole. While we may disagree with much that has been said, we do recognize that Elsevier has not done a good job communicating what we do and how we support both the peer-review process and the dissemination of work. In particular, we have left authors, editors, reviewers, and board members with the impression that we are focussed on restricting access rather than making their research as widely available as possible.

Helping editors, authors, reviewers, and board members to work easily on journals is central to us. We have systems in place to make it easier for editors to run large journals, some of which are dealing with thousands of submissions each yearsomething which most smaller publishers are not equipped to do. There will, of course, be people 
who argue against the involvement of privately owned organizations in academic publishing, but we believe that a mixed economy brings benefits to mathematics.

Professor Gowers's protest is specifically concerned with three issues: the pricing of journals; the practice of offering journals in large "bundles"; and, in particular, Elsevier's support, along with others, of a set of legislation, including the Research Works Act, in the United States.

\section{Pricing}

Mathematics journals published by Elsevier tend to be large, with a great many articles published each year. On a price-per-article or price-per-page level, our prices are typically, but not always, lower than those of other mathematics publishers. Our average price increase over the last eight years has been in the lowest quartile of the sector. The Cost of Knowledge statement selects ten (of thirty-eight) Elsevier journals to quote an average 2007 price per page of $\$ 1.30$ and compares this to prices per page ranging from $\$ 0.13$ to $\$ 1.21$ for selected journals from other publishers. However, the average price per page for all thirty-eight Elsevier journals in the AMS dataset for that year is $\$ 0.76$ per page, with several below $\$ 0.50$ per page and as low as $\$ 0.35$ per page. This is below the average for all mathematics journals in the AMS dataset. The document mentions that seven of the top ten most expensive journals are from Elsevier but does not show that the average price per page for those seven Elsevier journals is \$0.61.

That said, these figures are five years old, and in recent years we have made moves to reduce or freeze the prices of a number of our mathematics titles, recognizing that this field is not well funded and the articles are used intensively rather than frequently.

Journals such as the Journal of Algebra, Topology and its Applications and the Journal of Number Theory have all seen price reductions in recent years. Our target is for all of our core mathematics titles to be at or below US\$11 per article $^{1}$ (equivalent to $\$ 0.50-\$ 0.60$ cents per normal typeset page) by next year, placing us below most university presses, some societies, and all other commercial competitors. That will lead to a number of our titles seeing further and significant price reductions in their next volumes.

\section{"Bundling"}

Most journals are subscribed to as part of large deals or national consortia agreements, and so universities receive access to many more journal titles than they individually subscribe to and thus pay less than the list price described above. Although such packages are offered by virtually all

\footnotetext{
${ }^{1}$ This is calculated by dividing the institutional list price of a journal by the number of articles puclished.
}

publishers, many mathematicians have expressed dislike for such policies.

To describe this concisely, such agreements involve universities maintaining a core holding of journals and then, depending upon the size of the institution, having the option to subscribe to subcollections, such as in mathematics, or to all of our titles at a discount of the normal journal subscription list price. These collections can be as low as 2.5 percent of the catalogue value of the collection, which is one of the reasons why they have been so popular. A similar arrangement allows national consortia of universities to share electronic access to all their subscribed journals amongst themselves, without each university needing to hold an individual subscription.

We therefore disagree with the term "bundling", as it is not mandatory for a customer to enter into such a large deal. Libraries can decide what they want to subscribe to, whether that is individual titles or individual titles within a collection, or to join a national consortium. We do recognize the wish for more choice and flexibility, especially within departments, and we are currently experimenting with new ways of doing this. But switching off such schemes would, in our view, have a detrimental impact on access to the research literature. Because of the introduction of such large agreements, coupled with the simultaneous organization of universities and libraries into large consortia, access to journal content has never been better. However, clearly there are still areas and occasions where access is not at the level where it should be, and we are determined to address this issue.

\section{Access}

Later in this response we describe the major new steps that we are taking to ensure substantially wider free access to the mathematics articles that Elsevier publishes, but we'd like to flag two other issues that we think are misunderstood.

First, all of our titles, including our mathematics titles, are available in the poorest countries. We are a founding partner in Research4Life, a public/ private partnership providing journal content to researchers in the developing world. More than 2,000 Elsevier journals and 6,000 Elsevier e-books are available through Research4Life.

Second, authors in mathematics can post their author manuscripts, including corrections made in peer review and editing, on the arXiv. We have been allowing this for years. Elsevier supplies metadata directly to the arXiv and has for many years. There seems to be some misunderstanding on this point, as Elsevier's policies are no different from other major mathematics publishers in this regard, and we are happy to be able to correct this point. See our article posting 
policies (http://www.elsevier.com/wps/ find/authorsview.authors/postingpolicy) for more information.

\section{U.S. Legislation}

We are conscious that much of what triggered Professor Gowers's original posting was the support that Elsevier, along with others, gave to proposed U.S. legislation concerned with state mandates for publishing final versions of articles.

Almost all publishers are uncomfortable about laws determining what's published and under what conditions, but the critical feedback on this issue has been very sobering for many of us and has led to much reflection within the company.

That is why Elsevier announced that we are withdrawing our support for the Research Works Act.

\section{Quality of Journals}

The discussion about publishing and large subscription agreements has also highlighted concerns about the quality of particular journals. In the specific case of Chaos, Solitons and Fractals, this is a journal which has been rebuilt, with a new set of editors-whose work and commitment we would like to acknowledge-and much involvement from in-house Elsevier staff. We think that we did the right thing to seek to rebuild this journal. This journal has changed and informed the way in which we work on many journals, from our basic editorial contracts with editors to the use of much more formal editorial processes, electronic peer review, clearer statements on ethical issues, and the introduction of additional staff in support of journals.

We have put a great deal of effort in recent years into developing our support for journals, including significant editorial changes. Publishing judgment can go wrong and that will happen in any organization. But we learn from our mistakes and make considerable efforts to address and resolve them.

We do, however, need to be more open about the actions that we have taken when things go wrong. Specifically, we need to develop a better forum for listening to the mathematics community, to hearing specific criticisms, and to jointly developing policies and to hearing critical feedback.

\section{How Elsevier Is Responding to Feedback from the Community}

In this article we have responded to some of the concerns raised, but our goal is to do what it takes to ensure that the leading mathematics journals that we publish are as valuable and respected — and contribute as much back to the community-as any journal published by a society or university press. We are therefore taking the following steps now:

1. To make clear our commitment to wider access, we have made the archives of over forty core mathematics journals open for free, from four years after publication back to 1995, the year when we started publishing digitally;

2. On subscription pricing, we will target a price of US\$10-US\$11 per article (equivalent to \$0.50$\$ 0.60$ cent a page) for our core mathematics journals, below that of most of our competitors.

3. On "bundling", we are open to engaging with the mathematics community on how the system could work better, with greater flexibility and choice, and especially for small institutions without access to wider institutional resources. As a first step we are defining a smaller subcollection of core mathematics titles.

4. We will create an advisory scientific council for mathematics to ensure that we are working in tandem with the mathematics community to address feedback and to give greater control and transparency to the community.

5. We have announced that we are withdrawing our support for the Research Works Act.

We do not regard this as the end of our discussion with mathematicians, but rather as the continuation of our efforts.

We are very open to talking with anyone in the community about what we do and how we do it. Some fair criticism has come to us, which we will address.

More generally, we seek out and welcome the views of any concerned member of the mathematics community. 